\title{
Is pleural effusion in COVID-19 interstitial pneumonia related to in-hospital mortality?
}

\author{
Alberto Cereda, ${ }^{1}$ Marco Toselli, ${ }^{1}$ Anna Palmisano, ${ }^{2}$ Riccardo Leone, ${ }^{2}$ Davide Vignale, ${ }^{2}$ Valeria Nicoletti, ${ }^{2}$ \\ Gianluca Campo, ${ }^{3}$ Alberto Monello, ${ }^{4}$ Davide Ippolito, ${ }^{5,6}$ Francesco Giannini, ${ }^{1}$ Antonio Esposito ${ }^{2}$ \\ ${ }^{1}$ Interventional Cardiology Unit, GVM Care and Research Maria Cecilia Hospital, Cotignola (RA); ${ }^{2}$ Experimental Imaging \\ Center, IRCCS San Raffaele Hospital, Milan; ${ }^{3}$ Cardiovascular Institute, University Hospital of Ferrara, Cona (FE); ${ }^{4}$ Department \\ of Cardiology, Guglielmo da Saliceto Hospital, Piacenza; ${ }^{5}$ Department of Diagnostic Radiology San Gerardo Hospital, Monza; \\ ${ }^{6}$ Department of Medicine and Surgery, University of Milan-Bicocca, Monza, Italy
}

\begin{abstract}
The recent severe acute respiratory syndrome-related coronavirus 2 (SARS-CoV-2) pandemic has highlighted the importance of pulmonary computed tomography (CT) for diagnosis and prognostic stratification of this new viral pneumonia. 1370 lung CT scans (performed at the time of admission) of consecutive patients hospitalized for SARS-CoV-2 in Northern Italy during the first epidemic wave were analyzed by a radiological CoreLab. The presence of pleural effusion on pulmonary CT scan was present in 188 patients (13.3\% of the population) and identified a population with more comorbidities. Patients with pleural effusion had more cardio-respiratory complications with higher mortality. Pleural effusion was an independent predictor of death on multivariate analysis with an HR of 1.4 (95\% confidence interval 1-1.9). Pulmonary CT pleural effusion was an independent predictor of mortality.
\end{abstract}

\section{Introduction}

Coronavirus disease 2019 (COVID-19), caused by infection from severe acute respiratory syndrome-related coronavirus 2 , has spread worldwide. It causes a respiratory illness ranging from subclinical disease to severe interstitial pneumonia requiring orotracheal intubation and mechanical ventilation.

Correspondence: Alberto Cereda, Interventional Cardiology Unit, GVM Care \& Research Maria Cecilia Hospital, via Corriera 1, 48033 Cotignola (RA), Italy.

E-mail: tskcer@hotmail.it

Key words: COVID-19; chest; computed tomography; pneumonia; pleuric effusion.

Contributions: the authors contributed equally.

Conflict of interests: the authors declare no potential conflict of interests.

Availability of data and materials: data are available upon request.

Ethics approval and consent to participate: the study was approved by the Emilia Romagna regional ethics committee. Individual participating hospitals further approved the study.

Received for publication: 2 December 2020.

Accepted for publication: 3 December 2020.

This work is licensed under a Creative Commons Attribution NonCommercial 4.0 License (CC BY-NC 4.0).

${ }^{\circ}$ Copyright: the Author(s), 2021

Licensee PAGEPress, Italy

Italian Journal of Medicine 2021; 15:56-58

doi:10.4081/itjm.2021.1440
The chest computer tomography (CT) at the hospital admission allows COVID-19 interstitial pneumonia diagnosis and its severity assessment.

The CT features have an evolution pattern, including ground-glass opacity, consolidation, interlobular septal thickening, adjacent pleural thickening, and airbronchograms. ${ }^{1}$ The pneumonia is most commonly bilateral, involving the lower lobes.

Pleural effusion, pericardial effusion, lymphadenopathy, and pneumothorax are some of the uncommon but possible findings observed with disease progression. Pleural effusion (PE) has been reported in $5-7 \%$ of the cases, with a higher prevalence in patients with more severe lung involvement. ${ }^{2,3}$

The present study aims to evaluate the role of pleural effusion, as detected by the admission chest $\mathrm{CT}$, in predicting mortality of patients hospitalized for COVID-19.

\section{Materials and Methods}

A total of 1370 consecutive patients with a laboratory-confirmed COVID-19 infection who underwent chest $\mathrm{CT}$ at hospital admission were enrolled.

Clinical, laboratory and radiological data were collected from the participating centers, and CTs were analyzed by an expert cardiothoracic radiologist of a Core-lab blinded to patients' outcomes.

Demographics and comorbidities, including hypertension, diabetes, coronary artery disease, chronic lung disease, chronic kidney disease, and malignancy, were reported. Baseline hemoglobin, white blood cell count, creatinine, lactate dehydrogenase, and C-reac- 
tive protein (CRP) were analyzed. Lung well-aerated volume, pneumonia extension (scored as lung involvement of $25 \%, 25-50 \%, 50-75 \%,>75 \%$ ), pleural and pericardial effusion were evaluated at the CT.

In-hospital outcomes were categorized as hospitalization with non-invasive ventilation, orotracheal intubation, mechanical ventilation, or in-hospital death. Time interval from admission to death event was also reported. Acute coronary syndrome, pulmonary embolism, and cerebral stroke were defined as major cerebrovascular complications during hospitalization. The population was divided into two subgroups: patients experiencing interstitial pneumonia with PE and those without PE. T-student was applied for the quantitative variables and the chi-square for the qualitative variables. Statistically significant variables at the univariate were included in the multivariate analysis (Cox-regression) using time from hospital admission to the death event.

\section{Results and Discussion}

All the patients (mean age $67 \pm 13$ years; $68.8 \%$ male) had a radiological diagnosis of pneumonia. A total of $188(13.7 \%)$ subjects had a pleural effusion at baseline CT with bilateral distributions in $59.8 \%$ of patients. Patients with pleural effusion were older ( $72 \pm 12$ years $v s 66 \pm 13$ years, $\mathrm{P}=0.001$ ), with a slightly more significant burden of comorbidities (hypertension, diabetes, chronic lung disease, chronic kidney disease, coronary artery disease, and malignancy) (Table 1).

Compared to patients without PE, PE patients had lower baseline hemoglobin levels (12.2 $\pm 1.9 v s$ $13.6 \pm 1.8 \mathrm{~g} / \mathrm{dL}, \mathrm{P}=0.001)$ and higher $\mathrm{CRP}$ values (17.8 \pm 14 vs $13.8 \pm 12 \mathrm{mg} / \mathrm{dL}, \mathrm{P}=0.001)$. Patients with $\mathrm{PE}$ had a lower aerated lung volume $(1709 \pm 1076 v s$ $2521 \pm 1388 \mathrm{~mL}, \mathrm{P}=0.001)$ and a greater lung involvement ( $\geq 50 \%, 42.0 \%$ vs $17.7 \%, \mathrm{P}=0.001) .14 .9 \%$ of

Table 1. Demographical, clinical, radiological variables and outcomes of patients with and without pleural effusion diagnosed at pulmonary computed tomograpy at the time of hospitalization.

\begin{tabular}{|c|c|c|c|c|}
\hline Variables & All patients & Patients with PE & Patients without PE & P-value \\
\hline \multicolumn{5}{|l|}{ Population demographics } \\
\hline No. patients, N (\%) & 1370 & $188(13.7)$ & $1182(86.3)$ & - \\
\hline Age, years $\pm S D$ & $67 \pm 13$ & $72 \pm 12$ & $66.5 \pm 13$ & 0.001 \\
\hline Female sex $(\%)$ & 31.2 & 33 & 30.9 & 0.56 \\
\hline \multicolumn{5}{|l|}{ Clinical history } \\
\hline Hypertension (\%) & 56.9 & 58.3 & 56.6 & 0.66 \\
\hline Diabetes $(\%)$ & 19.3 & 21.4 & 18.9 & 0.43 \\
\hline Coronary artery disease $(\%)$ & 8.5 & 9.6 & 8.2 & 0.57 \\
\hline Chronic lung disease (\%) & 10.6 & 15.1 & 9.9 & 0.029 \\
\hline History of oncological malignancy (\%) & 5.6 & 15.1 & 4 & 0.001 \\
\hline \multicolumn{5}{|l|}{ Laboratory variables } \\
\hline Hemoglobin, $\mathrm{g} / \mathrm{dL} \pm \mathrm{SD}$ & $13.4 \pm 1.8$ & $12.2 \pm 1.9$ & $13.6 \pm 1.8$ & 0.001 \\
\hline Creatinine, $\mathrm{mg} / \mathrm{dL} \pm \mathrm{SD}$ & $1.1 \pm 0.4$ & $1.16 \pm 0.51$ & $1.08 \pm 0.4$ & 0.028 \\
\hline $\mathrm{LDH}, \mathrm{U} / \mathrm{L} \pm \mathrm{SD}$ & $389 \pm 157$ & $390 \pm 156$ & $382 \pm 165$ & 0.56 \\
\hline $\mathrm{CRP}, \mathrm{mg} / \mathrm{dL} \pm \mathrm{SD}$ & $14.4 \pm 12$ & $17.8 \pm 14$ & $13.8 \pm 12$ & 0.001 \\
\hline \multicolumn{5}{|l|}{ Chest computed tomographic radiological variables } \\
\hline Presence of coronary calcification $(\%)$ & 58.5 & 61.5 & 58 & 0.35 \\
\hline Aerated lung volume, $\mathrm{mL} \pm \mathrm{SD}$ & $2405 \pm 1378$ & $1709 \pm 1076$ & $2521 \pm 1388$ & 0.001 \\
\hline Interstitial lung involvement $<25 \%$ & 30.4 & 20 & 32.1 & 0.001 \\
\hline Interstitial lung involvement $25-50 \%$ & 42.7 & 43.7 & 36.4 & 0.001 \\
\hline Interstitial lung involvement $50-75 \%$ & 18 & 32.8 & 15.5 & 0.001 \\
\hline Interstitial lung involvement $>75 \%$ & 3.1 & 9.2 & 2.2 & 0.001 \\
\hline Right pleuric effusion $(\%)$ & 10.9 & 76.4 & - & - \\
\hline Left pleuric effusion $(\%)$ & 11.8 & 83.1 & - & - \\
\hline Bilateral pleuric effusion $(\%)$ & 8.5 & 59.8 & - & - \\
\hline Pericardial effusion (\%) & 5.6 & 14.9 & 4.1 & 0.001 \\
\hline Pleuro-pericardial effusion (\%) & 2.1 & 14.9 & 0 & 0.001 \\
\hline \multicolumn{5}{|l|}{ Hospital outcomes variables } \\
\hline Non-invasive ventilation without intubation $(\%)$ & 16.8 & 19 & 16.4 & 0.37 \\
\hline Mechanical invasive ventilation (\%) & 13.8 & 13.8 & 13.8 & 0.98 \\
\hline Time to death from hospital admission (days $\pm \mathrm{SD})$ & $11.5 \pm 11$ & $10.5 \pm 8.6$ & $12.5 \pm 11$ & 0.42 \\
\hline Acute coronary syndrome $(\%)$ & 0.8 & 2.1 & 0.5 & 0.019 \\
\hline Stroke $(\%)$ & 1 & 1.5 & 0.9 & $0, .43$ \\
\hline Pulmonary embolism (\%) & 3.6 & 7.7 & 2.9 & 0.001 \\
\hline Hospital mortality (\%) & 20.70 & 26.2 & 19.8 & 0.044 \\
\hline
\end{tabular}

PE, pleural effusion; SD, standard deviation; LDH, lactate dehydrogenase; CRP, C-reactive protein. 
the PE patients had a combined pleural-pericardial effusion. Of note, they also experienced a higher rate of acute coronary syndrome $(2.1 \%$ vs $0.5 \%$, $\mathrm{P}=0.0019)$ and pulmonary embolism $(7.7 \%$ vs $2.9 \%$, $\mathrm{P}=0.001$ ) during the hospitalization. No differences were detected in terms of orotracheal intubation between the sub-groups, but in-hospital mortality occurred more frequently in PE patients $(26.2 \% v s$ $19.8 \%, \mathrm{P}=0.044)$. In the Kaplan-Meier curve, the risk of 30-day death diverged significantly between the two groups (Log-rank 0.045) (Figure 1). In the multivariate Cox regression model, age, creatinine, interstitial involvement, and pleural effusion were independent predictors of 30-day death (Table 2). After the extent of lung involvement ( $>50 \%)$, the

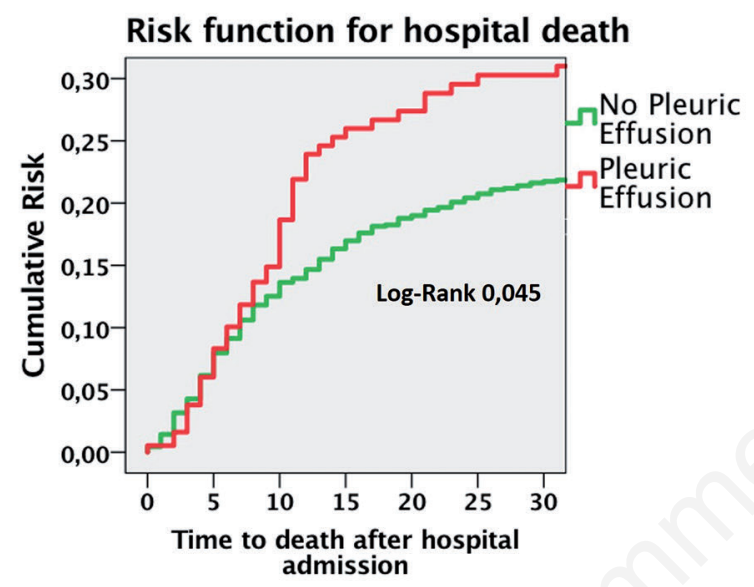

Figure 1. Kaplan-Meier for hospital mortality based on the presence or absence of pleural effusion to the thoracic computed tomographic scan performed upon admission to the hospital. The presence of pleural effusion was significantly associated with an increased risk of death in the first 30 days of hospitalization (Log-rank 0.045). presence of pleural effusion was the most significant categorical variable with an HR of 1.4 [95\% confidence interval (CI) 1.0-1.96]. Sex, basal reactive C protein, and clinical history (history of lung disease, diabetes mellitus, and history of cancer disease) were not statistically significant in the multivariate model. Basal creatinine was the most important quantitative predictor (HR 2.8 CI 95\% 2.2-3.6), more predictive than age.

\section{Conclusions}

Among patients with COVID-19-related interstitial pneumonia, PE is independently associated with higher in-hospital death. Accordingly, PE may help clinicians better identify COVID-19 patients at higher risk of poor outcomes, thus allowing healthcare resources optimization. The diagnosis of pleural effusion can be easily made through physical examination and using ultrasound and/or CT.

The diagnosis of a pleural effusion (clinical, ultrasound, or radiological) in the early stages of COVID-19 pneumonia must be a warning sign for clinicians and suggests more prompt monitoring and treatment of these patients at greater risk of adverse outcomes.

\section{References}

1. Bao C, Liu X, Zhang H, et al. Coronavirus disease 2019 (COVID19) CT findings: a systematic review and metaanalysis. J Am Coll Radiol 2020;17:701-9.

2. Li K, Wu J, Wu F, et al. The clinical and chest CT features associated with severe and critical COVID-19 pneumonia. Investig Radiol 2020;55:327-31.

3. Adams HJA, Kwee TC, Yakar D, et al. Chest CT imaging signature of COVID-19 infection. Chest 2020; S0012369220317335 [Epub ahead of print].

Table 2. Cox multivariate regression for age, creatinine, C-reactive protein, pulmonary interstitial radiological involvement $<\mathbf{5 0 \%}$, history of chronic lung disease and cancer, pulmonary embolism, pleural and pericardial effusion.

\begin{tabular}{lllcc}
\hline Baseline risk factors & Sign. & HR & $\mathbf{9 5 \%}$ CI lower & 95\% CI upper \\
\hline Age (years) & 0.000 & 1.077 & 1.063 & 1.091 \\
\hline Sex & 0.093 & 1.289 & 0.959 & 1.732 \\
\hline Creatinine (mg/dL) & 0.000 & 2.825 & 2.210 & 3.611 \\
\hline C-reactive protein (mg/dL) & 0.725 & 1.002 & 0.992 & 1.011 \\
\hline Lung interstitial involvement $>50 \%$ & 0.000 & 2.588 & 1.861 & 3.599 \\
\hline Presence of pleuric effusion & 0.047 & 1.406 & 1.005 & 1.967 \\
\hline History of lung disease & 0.605 & 1.092 & 0.783 & 1.521 \\
\hline Diabetes mellitus & 0.519 & 1.096 & 0.830 & 1.448 \\
\hline History of malignancy & 0.861 & 0.957 & 0.587 & 1.560 \\
\hline
\end{tabular}

